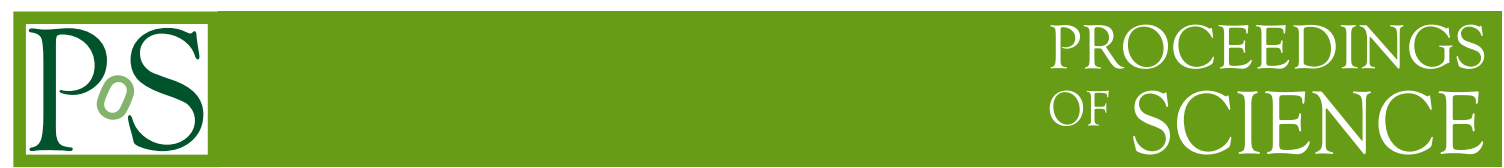

\title{
The Forward Search Experiment
}

\section{Di Wang* and Candan Dozen, Xin Chen, Zhen Hu}

Department of Physics, Tsinghua University, Shuangqing Road, Beijing 100084, China

E-mail: di.wang@cern.ch, zhen.hu@cern.ch

\begin{abstract}
Although the Standard Model successfully explains most phenomena at the LHC, there are several outstanding questions, including the nature of dark matter, the origin of neutrino masses, and the asymmetry in matter and anti-matter abundances in the Universe. Located in the side tunnel TI12, the Forward Search Experiment (FASER) will search for highly displaced signals from light and extremely weakly interacting particles that can be copiously produced in proton-proton collisions at the LHC. After their production at the ATLAS interaction point, light long-lived particles move along the beam collision axis line of sight, and then may decay within the volume of FASER into visible Standard Model particles.

During the current long shutdown, the FASER experiment will complete the hardware and software module production and commissioning and be installed underground. In Run 3 during 2021-2024, the FASER detector will start taking data.

Tsinghua University team produced the Tracker Interlock and Monitoring (TIM) board and the MPOD Interlock (MPODI) board to monitor the condition of the FASER tracker stations. If the temperature exceeds the normal range, the TIM board will send a hardware interlock signal to the LV and HV power supply, and the power supply will be turned off to protect the tracker station. Temperature and humidity data read from TIM will also be sent to DCS for further processing. MPODI monitors the status of chiller and controls the MPOD.
\end{abstract}

The Eighth Annual Conference on Large Hadron Collider Physics-LHCP2020

25-30 May, 2020

online

${ }^{*}$ Speaker 


\section{FASER Experiment}

The Forward Search Experiment (FASER) is a new experiment designed to search for undiscovered new physics at the LHC [1]. The FASER Collaboration consists of 64 members from 18 institutions and 8 countries [2]. The physics goal of the FASER experiment is to search for light and extremely weakly interacting particles, such as dark photons and axion-like particles, in proton-proton collisions at the LHC. These new particles are related to many important and basic questions, such as dark matter and anti-matter abundances. Long-Lived particles (LLPs) will be created near the ATLAS interaction point and then travel along the beam collision axis line of sight for $480 \mathrm{~m}$, and some of them will decay inside the FASER detector as shown in Fig. 1.

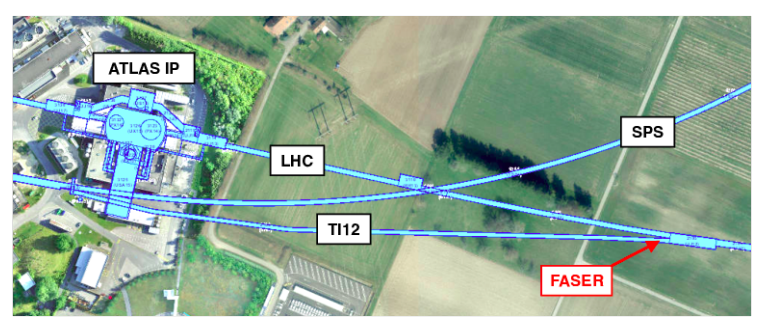

Figure 1: FASER detector location

The FASER detector is a small detector composed of scintillators, a decay volume, a spectrometer, tracker stations and an electromagnetic calorimeter, as shown in Fig. 2. The radius of the FASER main detector is $0.1 \mathrm{~m}$ and the total length is $4 \mathrm{~m}$. Located in the TI12 side tunnel, LLPs will arrive at the FASER main detector and decay into visible Standard Model particles. Besides the main detector, there is a subdetector named FASER $v$ [3], which will study neutrinos produced at the LHC. The FASER experiment will complete software and hardware production and commissioning and be installed during the current long shutdown. Data taking will start in LHC Run 3.



Figure 2: FASER detector components

\section{Tracker Interlock and Monitoring (TIM)}

The Tracker Interlock and Monitoring (TIM) module designed and fabricated by Tsinghua University team is the core of the interlock system of the FASER tracker. The main functions of the TIM boards are collecting temperature and humidity information from the tracker, sending all 
information to DCS for further processing, and hardware interlock based on temperature. Three TIM boards are installed to monitor the status of the three tracker stations. A fourth TIM board is used for FASER $v$ detector. These 4 boards together with an extra backup board are all contained in a TIM box, as illustrated in Fig. 3. The box will be fixed on the upper frame of the mini-rack.

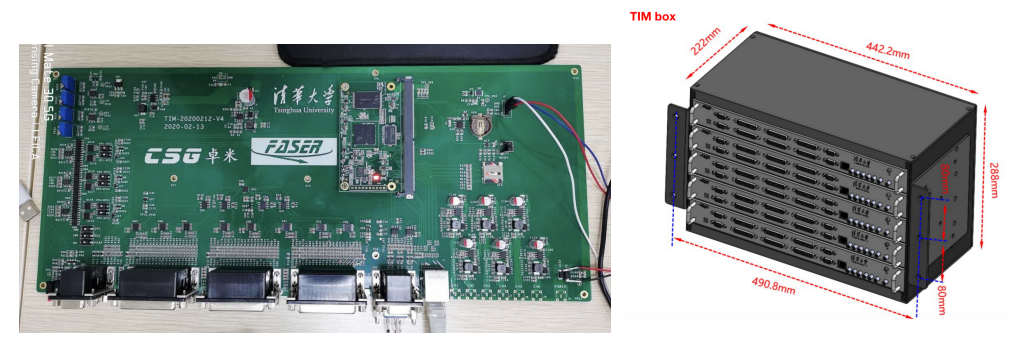

Figure 3: TIM board and TIM box

\section{MPOD Interlock (MPODI)}

The MPOD Interlock (MPODI) board, as shown in Fig. 4 is another important component of the tracker interlock system. The MPOD provides high voltage and low voltage to the FASER tracker system. There are two chillers that absorb harmful heat produced by tracker planes. The function of the MPODI board is to turn the MPOD off when two chillers stop working to avoid possible damages due to high temperature. As long as one chiller is working well, all channels of the MPOD will be kept in the normal working status. When both chillers stop working, all channels of the MPOD will be switched off with their specific down ramp.

\section{24V/6V Distributor}

The 24V/6V distributor board in Fig. 5 is part of the tracker power supply system. The function of this board is to provide $24 \mathrm{~V}$ and $1 \mathrm{~A}$ power for the TIM board and $6 \mathrm{~V}$ and $0.3 \mathrm{~A}$ power for $6 \mathrm{Patch}$ Panels. In total we need three $24 \mathrm{~V} / 6 \mathrm{~V}$ distributor boards for the three tracker stations and another one for the TIM board to monitor the ambient temperature.

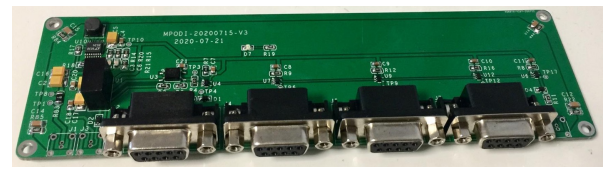

Figure 4: MPODI

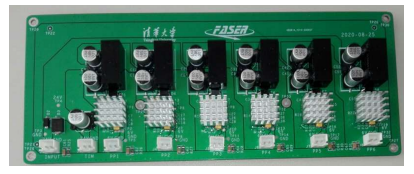

Figure 5: 24V/6V distributor

\section{References}

[1] FASER Collaboration, A. Ariga et al., [arXiv:1812.09139 [physics.ins-det]].

[2] FASER public webpage, https://faser.web.cern.ch.

[3] FASER Collaboration, H. Abreu et al., [arXiv:2001.03073 [physics.ins-det]]. 